\title{
Notes on the disentangling of spectra
}

\section{Enhancement in precision}

\author{
P. Hadrava \\ Astronomical Institute, Academy of Sciences, Boční II 1401, 14131 Praha 4, Czech Republic \\ e-mail: had@sunstel.asu.cas.cz \\ Received 15 August 2008 / Accepted 16 October 2008 \\ ABSTRACT

\begin{abstract}
Context. The technique of disentangling has been applied to numerous high-precision studies of spectroscopic binaries and multiple stars. Although, its possibilities have not yet been fully understood and exploited.

Aims. Theoretical background aspects of the method, its latest improvements and hints for its use in practice are explained in this series of papers.

Methods. In this first paper of the series, we discuss spectral-resolution limitations due to a discrete representation of the observed spectra and introduce a new method how to achieve a precision higher than the step of input-data binning.

Results. Based on this principle, the latest version of the KOREL code for Fourier disentangling achieves an increase in precision for an order of magnitude.
\end{abstract}

Key words. line: profiles - techniques: spectroscopic - stars: binaries: spectroscopic - methods: numerical

\section{Introduction}

Disentangling spectra of binary and multiple stars enables us to determine efficiently the orbital parameters and simultaneously to separate the spectra of the component stars. This numerical technique performed in either the wavelength domain (Simon \& Sturm 1994) or its Fourier image (Hadrava 1995) has been applied successfully in numerous studies of individual stellar systems. However, some users failed in their attempts or were unable to take full advantage of the method, partly due to a misunderstanding of its principles. A review of the Fourier disentangling has been provided by Hadrava (2004) together with the release in 2004 of the author's code KOREL, but, regarding new improvements of the method, this review is already out of date. The purpose of the present series of papers is to explain some common mistakes, provide practical hints for using the method, and present its new developments.

In this paper, consequences of the discretization of the observed spectra are discussed in Sect. 2. A new method for enhancement of the spectral resolution in disentangling spectra is introduced in Sect. 3. Results and their implications are briefly summarized in Sect. 4.

\section{Sampling of the input spectra}

In their study of disentangling, Hensberge et al. (2008) speculated about "expense" at which the computational efficiency of the spectral method dealing with the Fourier image surpasses the method of singular-value decomposition in the wavelength domain. They suggested that, among other things, it may be the need of having the input observed spectra sampled on a common grid equidistant in the logarithmic wavelength scale

$x=c \ln \lambda / \lambda_{0}$, where $\lambda_{0}$ is an arbitrarily chosen reference wavelength. It should be noted that the same assumption is commonly imposed on the solution in the wavelength domain as well, as it has been described by Simon \& Sturm (1994) and it is also obvious from the explanation in Fig. 1 of Hensberge et al. (2008) or their example in Appendix of the same paper.

The uniform sampling of input data simplifies the solution, but this assumption may be avoided in both methods of disentangling. In the Fourier view, it is obvious that the Fourier transforms $\widetilde{I}(y)$ of the observed spectra $I(x)$ in chosen (equidistant) sampling frequencies $y_{k}$ may be calculated directly according to the definition

$\tilde{I}\left(y_{k}\right)=\int I(x) \exp \left(i y_{k} x\right) \mathrm{d} x$

from any original (even non-equidistant) binning $x_{l}$ if the function $I(x)$ is suitably interpolated, e.g. by the simple linear formula

$I(x)=I\left(x_{l}\right) \frac{x_{l+1}-x}{x_{l+1}-x_{l}}+I\left(x_{l+1}\right) \frac{x-x_{l}}{x_{l+1}-x_{l}} \quad$ for $x \in\left(x_{l}, x_{l+1}\right)$.

The common practice of interpolating $I(x)$ first to the equidistant grid points and then using the Fast Fourier Transform saves the computer time (at some expense of accuracy), but is not inevitable in Fourier disentangling.

In the wavelength-domain solution, the single off-diagonal matrices ( $N$ in notation of Simon \& Sturm 1994) shifting the spectra of component stars to their appropriate positions in individual exposures may be replaced by wider band matrices if the observed spectra are not sampled in the same equidistant set of the logarithmic wavelengths into which the component spectra are to be separated (Simon \& Sturm 1994, p. 287: "The submatrices of $\boldsymbol{M}, \boldsymbol{N}_{A i}$ and $\boldsymbol{N}_{B i}$, are rectangular band matrices with a bandwidth depending on the differences in dispersion of the wavelength scales of $\boldsymbol{c}$ and $\boldsymbol{x}$ "). A simple possibility is to use a 


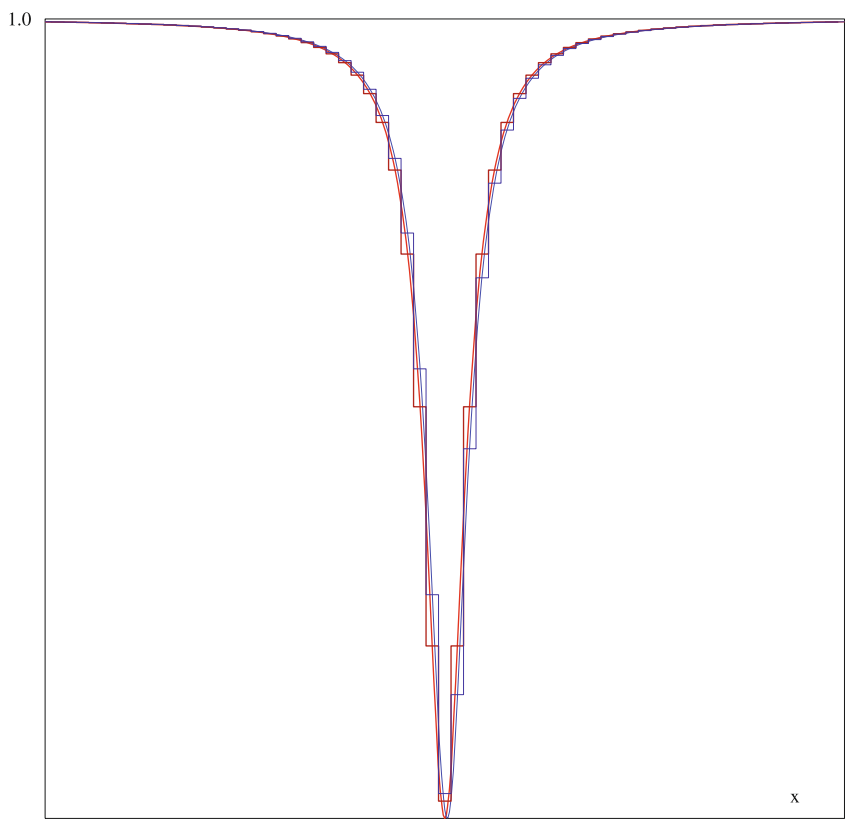

Fig. 1. Discretization of a Lorentzian profile $I(x)$ (the smooth thick line) centered on the pixel position should yield a symmetric distribution of counts $D[I](x)$ in neighbouring bins (the thick step function). A slightly shifted profile $I^{\prime}(x)$ (for 0.2 pixel-width in this figure - see the thin lines) results in an asymmetry of the counts $D\left[I^{\prime}\right](x)$, which in turn enables us to determine the line position at a precision below the pixel width.

matrix $N$ with two non-zero elements in each column given by Eq. (3). This is, however, again equivalent to a suitable resampling, and a subsequent solution in the convenient representation of the observed spectra. An additional significance of using bandwith matrices to refine the model is discussed in the next Section.

Although any resampling implies some smoothing of the input signal and thus a loss of information in the high-frequency modes, it is inevitable provided the observations are not directly obtained in the required data bins. The question is therefore not about performing a resampling, but how it can be performed best. This problem, which is common to any method of disentangling, is related to the more general task of optimal dataprocessing of observed spectra (cf. Hensberge 2004) and its aspects in disentangling will be studied in detail elsewhere.

A consequence of discretizing observed spectra is limitation of the accuracy at which the radial velocities are determined. Until now, a common practice in both the wavelength-domain and Fourier disentangling was to round the expected Doppler shift to an integer multiple of the radial-velocity step. This limited resolution of Doppler shifts in the individual spectra also limits the precision of the disentangled orbital elements and the sharpness of the separated spectra of component stars. A straightforward means of improvement appears to be a choice of a smaller sampling step. However, the resolution is limited by the detectors in any case. On the other hand, it is intuitively evident that if we have a set of spectra with mutually shifted sampling, we can also reconstruct details on a sub-pixel scale. For instance, if a very narrow line with a sub-pixel width moves from a given pixel in some exposures to the neighbouring pixel in other exposures, then its position may be found precisely from the time and its width from the duration of this transition. It is thus worth investigating the limits of resolution in details.

\section{An increase of spectral resolution}

In my spectral method, the shift in the spectra $I(x)$ of each component (which we wish to separate from the observed superpositions), in the logarithmic wavelength scale $x$ defined by Eq. (1) for a value $v$ of the radial velocity, is given by the convolution of the spectra with the shifted Dirac delta-function $\delta(x-v)$,

$I^{\prime}(x)=I(x-v)=I(x) * \delta(x-v)$,

which implies, in the Fourier transform $(x \rightarrow y)$, a multiplication by a function $\exp (i y v)$,

$\tilde{I}^{\prime}(y)=\tilde{I}(y) \exp (i y v)$

(cf. $I_{j}$ in Eqs. (1) and (2) of Hadrava 1995). This simple exponential function can be evaluated precisely at each frequency $y$. However, due to the limited number $N$ of the modes taken into account, its inverse Fourier transform will generally produce a wider peak with some ghosts on its sides resembling interference fringes. Only in the special case of $v$ being an integer multiple of the grid step, the period of function $\exp (i y v)$ is in resonance with the interval length in $y$-representation and a sharp shifted $\delta$ function coinciding with a grid point of the $x$-representation can be reproduced. For that reason the radial velocity was rounded to the nearest grid point in the Fourier disentangling also and it explains why the radial velocities or their residuals calculated by the original KOREL-code were quantized depending on the radial-velocity step.

However, owing to the resolution in the digitalized values of intensity read from individual detector pixels, the position of spectral lines wider than the sampling step can be deduced with an accuracy exceeding the step width (cf. Fig. 1). Alternatively to a convolution with the shifted $\delta$-function, a shift of a spectrum $I(x)$ for value $v$ can be expressed as a Taylor expansion

$I(x-v)=\sum_{j=0}^{\infty} \frac{1}{j !} I^{(j)}(x)(-v)^{j}$,

which usually converges rapidly for small values of $v$. In a discrete equidistant representation $x_{k}$ with the step $\Delta_{x}$, the first two derivatives can be approximated by finite differences

$I^{(1)}\left(x_{k}\right) \simeq \frac{1}{2 \Delta_{x}}\left(I\left(x_{k+1}\right)-I\left(x_{k-1}\right)\right)$,
$I^{(2)}\left(x_{k}\right) \simeq \frac{1}{\Delta_{x}^{2}}\left(I\left(x_{k+1}\right)-2 I\left(x_{k}\right)+I\left(x_{k-1}\right)\right)$.

Therefore in the vicinity of the grid point $x_{k}$ a small shift of $I$ can be expressed in terms of values in this and the two neighbouring points as

$$
\begin{aligned}
I\left(x_{k}-v\right) \simeq & I\left(x_{k}\right)-I^{(1)}\left(x_{k}\right) v+\frac{1}{2} I^{(2)}\left(x_{k}\right) v^{2}+o\left(v^{3}\right) \\
\simeq & I\left(x_{k}\right)-\frac{v}{2 \Delta_{x}}\left(I\left(x_{k+1}\right)-I\left(x_{k-1}\right)\right) \\
& +\frac{v^{2}}{2 \Delta_{x}^{2}}\left(I\left(x_{k+1}\right)-2 I\left(x_{k}\right)+I\left(x_{k-1}\right)\right)+o\left(v^{3}\right) .
\end{aligned}
$$

This implies that the operator $\delta(x-v)$ of the shift is approximated by

$$
\begin{aligned}
\delta(x-v) \simeq & \delta(x)-\frac{v}{2 \Delta_{x}}\left(\delta\left(x+\Delta_{x}\right)-\delta\left(x-\Delta_{x}\right)\right) \\
& +\frac{v^{2}}{2 \Delta_{x}^{2}}\left(\delta\left(x+\Delta_{x}\right)-2 \delta(x)+\delta\left(x-\Delta_{x}\right)\right)+o\left(v^{3}\right)
\end{aligned}
$$




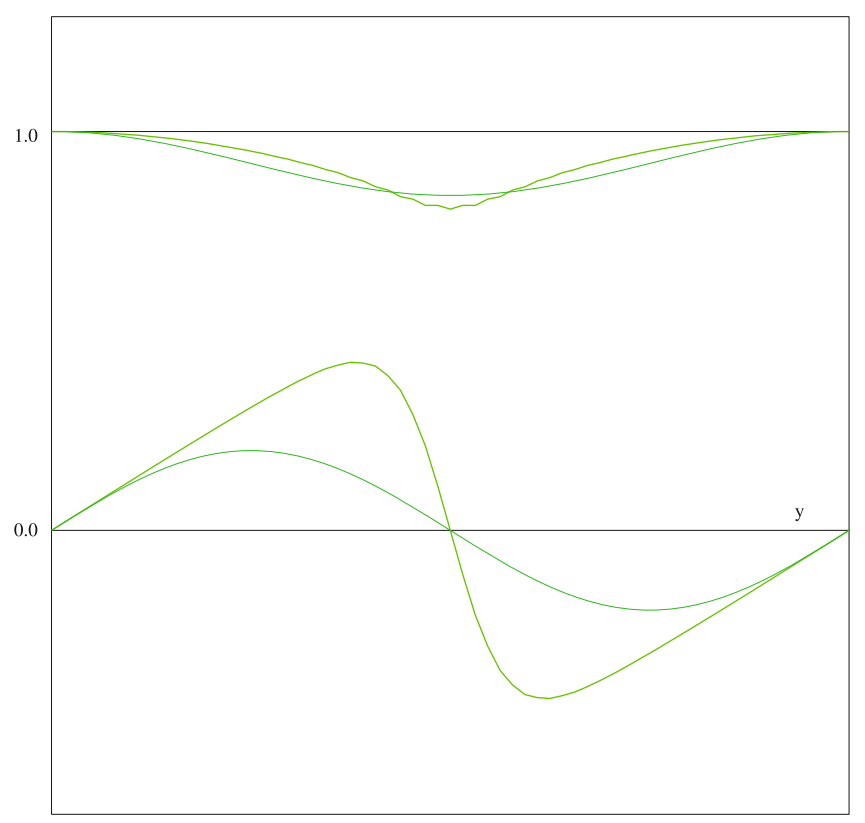

Fig. 2. Fourier transform of the discretized shifted profile $D\left[I^{\prime}\right]$ corresponds to the Fourier transform of the centered profile $D[I]$ multiplied by a correction, the real and imaginary parts of which are drawn by the upper and lower (distorted sinusoidal) thick lines, resp., while their approximations (11) are drawn by the thin sinusoidal lines.

and its Fourier transform

$$
\begin{aligned}
\exp (i y v) \simeq & 1-\frac{v}{2 \Delta_{x}}\left(\exp \left(-i y \Delta_{x}\right)-\exp \left(i y \Delta_{x}\right)\right) \\
& +\frac{v^{2}}{2 \Delta_{x}^{2}}\left(\exp \left(-i y \Delta_{x}\right)-2+\exp \left(i y \Delta_{x}\right)\right)+o\left(v^{3}\right) \\
& =1+\frac{i v}{\Delta_{x}} \sin \left(y \Delta_{x}\right)+\frac{v^{2}}{\Delta_{x}^{2}}\left(\cos \left(y \Delta_{x}\right)-1\right)+o\left(v^{3}\right) .
\end{aligned}
$$

It can be seen in Fig. 2 that a ratio of the two Fourier transforms of mutually shifted profiles is approximated well by this simple sinusoid for small values of $y$, while for the higher-frequency modes (drawn closer to the middle of the figure) higher harmonics contribute significantly. This is an obvious consequence of the fact that the approximations (7) and (8) of the derivatives are more accurate for the lower modes, which do not change significantly on the scale of $\Delta_{x}$. The exact shape of the shift operator depends on the spectrum to be shifted, unless $v$ is an integer multiple of $\Delta_{x}$. It means that the value of $v$ cannot be disentangled with unlimited precision from roughly sampled, unknown spectra. However, already the application of the correction (11) improves the precision of the disentangling significantly, and the accuracy could be even higher for disentangling constrained by a template spectrum.

The above described procedure of reconstructing component spectra from a large set of observations should not be confused with a simple interpolation (given e.g. by Eq. (3)) in a single observation or between grid points of some theoretical models. For instance, in model atmospheres, the dependent variable (e.g. the specific intensity) is calculated usually for chosen exact values of the independent variable (wavelength) from which it can be interpolated to other values or integrated over some regions of the variable. On the other hand, in true observations, the values read at individual detector pixels are the quantities integrated over some interval of the wavelength, which provide some constraint only on the inner distribution. Without any additional
Table 1. Comparison of the old and new KOREL solutions.

\begin{tabular}{llll}
\hline \hline Parameter & $N[\%]$ & KOREL04 & KOREL08 \\
\hline$S$ & 0 & 3.61 & 0.012 \\
& 0.5 & 4.98 & 1.22 \\
& 1. & 8.76 & 4.85 \\
$\Delta T_{0}$ [Period] & 2. & 23.59 & 19.33 \\
& 0 & $4.9 \times 10^{-4}$ & $4 . \times 10^{-9}$ \\
& 0.5 & $4.9 \times 10^{-4}$ & $6.6 \times 10^{-6}$ \\
& 1. & $4.9 \times 10^{-4}$ & $2.3 \times 10^{-4}$ \\
$\Delta K_{1}\left[\mathrm{~km} \mathrm{~s}^{-1}\right]$ & 2. & $7.2 \times 10^{-4}$ & $5.0 \times 10^{-4}$ \\
& 0 & 0.49 & 0.07 \\
& 0.5 & 0.49 & 0.03 \\
$\Delta q ;$ & 1. & 0.49 & 0.06 \\
& 2. & 0.33 & 0.18 \\
& 0 & 0.010 & 0.0005 \\
& 0.5 & 0.010 & 0.0002 \\
& 1. & 0.010 & 0.0014 \\
& 2. & 0.013 & 0.0040 \\
\hline
\end{tabular}

information, these values may be used in a single exposure as an estimate of the variable for the middle of the interval, while closer to its edges the value interpolated between the neighbouring bins is more appropriate. However, for a set of exposures, the information can be combined to reveal partly also the subpixel structure, or, using the above described procedure, to find subpixel mutual shifts between the exposures.

As an example, we show a result of disentangling of simulated data. Twenty spectra uniformly covering one period (which is taken to be a unit of time) of a double-line binary on circular orbit with chosen radial-velocity semi-amplitudes $K_{1}=$ $50 \mathrm{~km} \mathrm{~s}^{-1}$ for the primary, and $K_{2}=100 \mathrm{~km} \mathrm{~s}^{-1}$ for the secondary were calculated. For each component, one line with a Lorentzian profile (with central depths 0.3 and 0.2 of the common continuum and half-widths equal to 30 and $40 \mathrm{~km} \mathrm{~s}^{-1}$ for the primary and secondary, respectively) was included. A pseudo-random noise scaled to amplitudes $n=0 \%, 0.5 \%, 1 \%$, or $2 \%$ of the continuum level was added. The spectra were sampled by integrating in bins of width corresponding to $10 \mathrm{~km} \mathrm{~s}^{-1}$. The results of disentangling obtained using the KOREL code in its old version (KOREL04 released by the author in 2004) and in its new version (KOREL08) of enhanced precision, are compared in Table 1. In this table, $S$ denotes the integrated square of spectra residuals, and $\Delta T_{0}$ is the difference in units of the period between the solved epoch of periastron (defined by fixed periastron longitude) and its true value $T_{0}=0$ chosen for the simulation. Similarly, $\Delta K_{1}$ are the differences between the calculated and true radial-velocity semi-amplitudes of the primary and $\Delta q$ for the mass ratio $\left(q=M_{2} / M_{1}=K_{1} / K_{2}=0.5\right)$.

It can be seen from the results that the squares $S$ of the residuals consist of a part approximately proportional to the square $n^{2}$ of the noise, as can be supposed, but also an other additive, almost constant part, which is comparable to the $1 \%$ noise in the solution with the classical KOREL04, but is suppressed for at least two orders in the super-resolution KOREL08. This part is obviously due to the discrepancies between sampling of the component spectra in different exposures shifted by a noninteger multiple of the sampling step. This contribution depends on the shape of the spectrum and its importance on the level of the noise. This explains why in preliminary applications to real data the new method yielded significantly superior results in some cases, but only a negligible improvement in other cases. 
Similarly, the errors in orbital parameters have a part that increases with the noise and a noise-independent part, which is significantly smaller in the solution based on the new KOREL08.

\section{Conclusions}

The correction provided in Eq. (11) for the residual part of the radial velocities over an integer multiple of the sampling step improves the Fourier disentangling significantly. With this result and other improvements completed by the author to recent versions of the KOREL code, the version of 2004 is no longer supported, and we recommend using for true applications the version of 2008.

Due to the equivalence of Eqs. (4) and (5), the wavelengthdomain solution could be improved similarly if the single offdiagonal matrix $N$ would be replaced by a three- (off-)diagonal matrix (10), or even by a more complicated matrix, if it should also include an interpolation from a non-uniform sampling of the input data. A possibility for using band-matrices was mentioned by Simon \& Sturm (1994), and in more detail explained by Sturm (1994) (cf. also Hensberge et al. 2008).
The method described here could be improved to achieve an even higher precision for known component spectra (i.e. for the constrained disentangling), for which the higher harmonics of the approximation given by Eq. (11) could be estimated. Analogous numerical refinement either in direct or Fourier space could be useful also in other methods in spectroscopy (e.g. in methods using the broadening function) as well as in data processing in other fields of astrophysics.

Acknowledgements. This work has been completed in the framework of the Center for Theoretical Astrophysics (ref. LC06014) with a support of grant GAČR 202/06/0041. The comments by the referee Saša Ilijić are highly appreciated.

\section{References}

Hadrava, P. 1995, A\&AS, 114, 393

Hadrava, P. 2004, Publ. Astron. Inst. ASCR, 92, 15

Hensberge, H. 2004, ASP Conf. Ser., 318, 43

Hensberge, H., Ilijić, S., \& Torres, K. B. V. 2008, A\&A, 482, 1031

Simon, K. P., \& Sturm, E. 1994, A\&A, 281, 286

Sturm, E. 1994, Ph. D. Thesis, Ludwig-Maximilians-Universität, München 